\title{
Lesions Detection of Multiple Sclerosis in 3D Brian MR Images by Using Artificial Immune Systems and Support Vector Machines
}

\author{
Amina Merzoug, Laboratoire SIMPA, USTO-MB, Bir El Djir, Algeria \\ Nacéra Benamrane, Laboratoire SIMPA, USTO-MB, Bir El Djir, Algeria \\ Abdelmalik Taleb-Ahmed, Polytechnic University of Hauts-de-France, Valenciennes, France
}

\begin{abstract}
This paper presents a segmentation method to detect multiple sclerosis (MS) lesions in brain MRI based on the artificial immune systems (AIS) and a support vector machines (SVM). In the first step, AIS is used to segment the three main brain tissues white matter, gray matter, and cerebrospinal fluid. Then the features were extracted and SVM is applied to detect the multiple sclerosis lesions based on SMO training algorithm. The experiments conducted on 3D brain MR images produce satisfying results.
\end{abstract}

\section{KEYWORDS}

3D Brain MRI, AIS, Detection, Lesions, Multiple Sclerosis, Segmentation, SMO, SVM

\section{INTRODUCTION}

Multiple sclerosis is an autoimmune chronic disease of the central nervous system especially the brain, the optic nerves and the spinal cord. The symptoms are very variable, numbness of a limb, blurred vision, loss of equilibrium...etc (Xavier et al, 2012).

Magnetic resonance (MR) imaging can accurately visualize and locate plaques in both the brain and spinal cord. Depending on the sequences used, they appear white (in technical terms, we speak of "hypersignals") or black ("hyposignals").

In 2019, more than 2.4 million people suffer from multiple sclerosis . The research is focused on finding innovative treatments to relieve people with MS. The goal of this study is to detect abnormalities of gray matter and white matter in MS from 3D RM Image

Many methods have been proposed to automatically segment lesions since manual segmentation requires expert knowledge, is time consuming and is subject to intra- and interexpert variability (Vera-Olmos et al, 2016).

Veronese et al (Veronese et al, 2013) proposed a fuzzy classification algorithm that uses spatial information for MS lesion segmentation. In addition to spatial information, standard deviation dependent filtering is incorporated into the algorithm to provide better noise immunity. Also, fuzzy logic is adjusted to be more selective on vertical elliptical objects instead of circular objects since most plates are in this form.

Saba et al (Saba et al, 2018) presented a method of segmentation of MS lesions beginning with contour detection using the canny algorithm, and then a modified blurred mean c algorithm is applied 
to increase the accuracy of the diagnosis. Pre-treatment techniques are applied to get the best result were used, such as the brain extraction tool and binarisation

Bassem (Bassem, 2012) proposed a technique for segmentation of Sclerosis lesions by using texture textural features and support vector machines. They used two generic configurable components: a central processing module that locates areas of the brain that may form MS lesions, and a postprocessing module that adds or removes these areas for more accurate data. Based on these configurable modules, single-view segmentation and multiple-section view pipelines are provided to address the limitations found in segmentation results.

Khotanlou et al (Khotanlou et al, 2011) proposed a SCPFCM algorithm named based on t membership, typicity and spatial information. Firstly, initial segmentation is applied to T1-w and T2-w images to detect MS lesions. then the non-cerebral tissues are removed by using morphological functions and finally for extraction of MS lesions, the result of the image T1 is used as a mask and compared to the image $\mathrm{T} 2$.

Ayelet et al (Ayelet et al, 2009) presented a multiscale method to detect lesions in multiple sclerosis based on two phases: segmentation and classification. The first one obtains a hierarchical decomposition of a multichannel anisotropic MRI scans and produces a set of features. These features are used in the second phase via a decision tree to detect lesions at all scales.

The authors find that the problem of MS lesions segmentation is still widely open especially for supervised automatic approaches. This motivates to propose an automatic approach for MS lesion detection that uses a supervised learning without an explicit expert intervention. The approach is based on AIS for brain tissue segmentation and SVM with SMO for lesions detection. A number of features to define vector types of specific lesions were calculated and these vector types were used as inputs for SVM.

This paper is organized as follows. In section 2, the researchers resent their proposed approach. Section 3 shows the obtained experimental results. Section 4 describes the comparison with a previous work and another proposed method. The final section provides the conclusion of this work.

\section{THE PROPOSED APPROACH}

Automatic segmentation of MS lesions is difficult, as indicated in the previous section due to the large variability of multiple sclerosis lesions. Lesions have deformable shapes, their texture and intensity can vary and their location can also vary from one patient to another. The researchers propose to apply a new segmentation workflow based on a voxel analysis.

The method consists of three steps (see Figure 1). For each 3D MR image, the AIS are applied for segmentation of the three main brain tissues white matter, gray matter and cerebrospinal fluid. The authors compute a number of features then the SVM is used for MS lesions segmentation only on the white matter since MS lesions are located in.

\section{Brain Tissue Segmentation}

The researchers started by segmenting the image of the brain MR in the three classes mentioned (grey matter, white matter and cerebrospinal fluid) using the AIS algorithm.

\section{Segmentation by Artificial Immune Systems (AIS)}

Artificial immune systems is a model that encompasses both, mathematics and biological principles, as the natural immune system offers interesting features like memory and learning that will be useful for solving problems (Tavana et al, 2016). 
Figure 1. Flowchart of the proposed approach for MS lesions segmentation

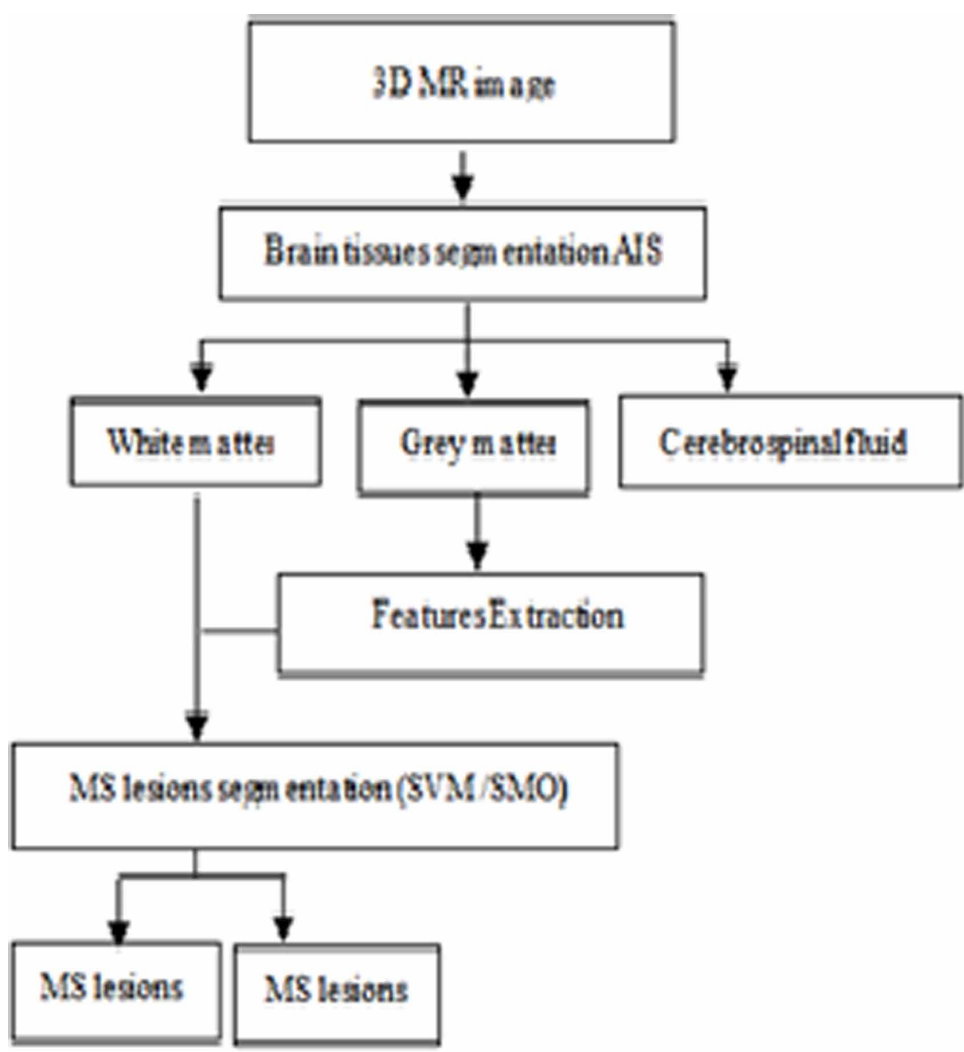

\section{Learning}

This phase is to find the memory cells (voxels) representing the regions in this study area and then make the classification using the algorithm of CLONCLAS that uses the principles of artificial clonal selection. The elements which are used in this algorithm are (Komaki et al, 2016):

- Antibody: or samples represents the basis of training that will recognize the image of antigens.

- Antigen: represents the basis of examples for which we want to determine the class.

- Affinity: affinity in immune systems is the measure of similarity between the antibody and the

- Antigen: the latter two are represented by a point. Affinity is the distance between these two points, in this work, the Euclidean distance is used.

- Memory cell: represents the best antibodies found for the class.

Learning occurs class by class by the CLONAG algorithm (Komaki et al, 2016):

1. Training samples are considered a priori to antibody $(\mathrm{Ab})$. One of these samples randomly drawn is likened to an antigen (Ag). Through the Euclidean distance, they calculated affinity of this Ag with all Abs of the class.

2. Abs voxels are ranked in descending order according to their affinity compared to the Ag considered. The first $\mathrm{N}$ voxels will be selected to undergo a cloning while preserving the first voxel to form the memory cell Mc class match. 
3. Clone the $\mathrm{n}$ selected voxels $\mathrm{i}$ in proportion to their affinity. The clones number of a voxel is even higher than the affinity of the voxel is high. This number is calculated as follows:

The number of clones for each member:

$\mathrm{Nc}=\operatorname{round}\left(\beta^{*}(\mathrm{n} / \mathrm{I})\right)$

With $\mathrm{Nc}$ is the number of clones of an element, $\beta$ is the cloning coefficient, I is the position of the element to be cloned, and round is a function that rounds a real number to an integer.

The total number of clones (Erik et al, 2012):

$$
N c=\sum_{i=0}^{n} \operatorname{round}\left(\beta *\left(\frac{n}{I}\right)\right)
$$

4. Mutate clones voxels. The mutation is the random change of the values of a voxel; it prevents the establishment of uniform individuals unable to evolve. Value must be inversely proportional to the affinity.

5. Calculate the affinity of all the new elements (clones and mutants) compared to Ag departure and classify them according to affinity.

6. If the affinity of the highest affinity element is greater than the affinity of the memory cell already selected, this item will become the new memory cell.

7. Replace departure training voxels with the lowest affinity, the best new evidence obtained.

8. Repeat steps 2 to 7 until the last Mc match memory cell is saved, which is the best Ab.

\section{Classification (Belmokhtar et al, 2012)}

After the completion of the learning process, they proceed to the classification thanks to memory cells. Indeed, each class of the image is now represented by its memory cell that is the best Ab representative of this class. The affinity is calculated for each voxel to be classified with respect to different memory cells. This voxel is assigned to the class that gives the best affinity. This phase comparison is made, a recognition phase ( $\mathrm{Ag}, \mathrm{Abs})$, because the voxel classification is an $\mathrm{Ag}$ which will be recognized by the $\mathrm{Ab}$ which is specific to it.

\section{Feature Extraction}

Various feature extraction methods has been proposed in the literature using first statistical texture features (Faro et al, 2010). As mentioned in a previous work (Merzoug et al, 2014), MS lesions appear hypo- or hyper-signal compared to the normal appearing white matter according to their type and MRI sequences. They define vector types of specific lesions.

In this study, for the T1-weighted images, a black hole corresponds to an intensity equivalent to the gray matter, or lower and for the T2-weighted images, an intensity corresponding to a hyper-signal compared to the white matter. Mathematically (Urien et al, 2016), the set of voxels i whose intensity $t_{i}$ satisfies is:

$$
t_{i}^{T 1} \leq \mu_{S G}^{T 1}+3 v_{S G}^{T 1}
$$


$\mu_{S B}^{T 2}+3 v_{S B}^{T 2} \leq t_{i}^{T 2}$

With:

$t_{i}^{X X}:$ Intensity in the image weighted at XX (T1 or T2) of the voxel i.

$\mu_{Y Y}^{X X}$ : computed mean of the tissue YY (gray matter or white matter) in the images weighted at $\mathrm{XX}$.

$v_{y y}^{X X}:$ computed variance of the tissue $\mathrm{YY}$ in the images weighted at $\mathrm{XX}$.

\section{The MS Lesions Segmentation}

For this step the authors used the machine vector support method (SVM). The intensity of multiple sclerosis lesions is close to the intensity of the gray matter or the intensity of the partial volume CSF / MG, to solve this problem they looked for lesions of MS only in the white matter. So they used a bi-class SVM (lesion of MS or no MS) with the RBF kernel (Belmokhtar et al, 2012).

\section{SVM Principle}

Support vector machines are supervised learning algorithms, used in many applications of pattern recognition, image processing, and Imaging medical (Chandan et al, 2018)

From a training set $\left(\vec{x}_{i}, y_{i}\right)$ where $\vec{x}_{i} \in R^{m}$ corresponds to datum and $y_{i}$ take value -1 or1 stands for the corresponding class, the goal of SVM is to identify the optimal separating hyperplane [Rode et al, 2012]:

$\vec{w} \cdot \vec{x}+b=0$

The function of classifier is defined by:

$f: \vec{x} \in R^{m} \rightarrow \operatorname{sign}(\vec{w} \cdot \vec{x}+b) \in\{ \pm 1\}$

With $\left\{\begin{array}{l}\vec{w} \cdot \vec{x}+b \geq 0 \text { si } y_{i}=+1 \\ \vec{w} \cdot \vec{x}+b \leq 0 \text { si } y_{i}=-1\end{array}\right.$

In addition, the optimal hyperplane separator must maximize the margin: $\frac{2}{\vec{w}}$. The solution is obtained by solving the problem of margin maximizing under the previous constraints, which leads to the following quadratic optimization problem:

$$
\min _{(w, b)} \frac{\vec{w}^{2}}{2}
$$

Such us: 
$y_{i}\left(\vec{w} \cdot \overrightarrow{x_{i}}+b\right) \geq 1, \forall i \in 1 \mid N$

The dual problem obtained by integrating the Lagrange multipliers is:

$$
\begin{aligned}
& \max _{\alpha} W(\alpha)=-\frac{1}{2} \sum_{i=1}^{N} \sum_{j=1}^{N} \alpha_{i} \alpha_{j} y_{i} y_{j} \overrightarrow{x_{i} x_{j}}+\sum_{i=1}^{N} \alpha_{i} \\
& \vec{w}=\sum_{i=1}^{N} \alpha_{i} y_{i} \vec{x}_{i} \text { and } b=y_{i}-\vec{w} \cdot \overrightarrow{x_{i}} \text { such as } \alpha_{i} \neq 0 \text {. The classification function is defined by: } \\
& f(\vec{x})=\vec{w} \cdot \vec{x}+b=\sum_{i=1}^{N} \alpha_{i} y_{i} \overrightarrow{x_{i}} \vec{x}+b
\end{aligned}
$$

They use variables relaxation $\xi_{i}$ and regularization parameter $\mathrm{C}$ to enlarge margin in nonlinearly separable data. The optimization problem becomes (Zidelmal et al, 2007):

$$
\min _{(w, b)} \frac{\vec{w}^{2}}{2}+c \sum_{i=1}^{N} \xi_{i}
$$

This optimization problem is solved using Lagrange multipliers and become:

$$
\max _{\alpha} W(\alpha)=-\frac{1}{2} \sum_{i=1}^{N} \sum_{j=i}^{N} \alpha_{i} \cdot \alpha_{j} \cdot y_{i} \cdot y_{j} \cdot \vec{x}_{i} \cdot \vec{x}_{j}+\sum_{i=1}^{N} \alpha_{i}
$$

The new problem is obtained by replacing the data set which vectors are only involved in the scalar product $\overrightarrow{x_{i}} \cdot \overrightarrow{x_{j}}$ by their counterparts in the new feature space $:\left(\overrightarrow{x_{i}}\right) \cdot\left(\overrightarrow{x_{j}}\right)$ (Locoeur et al, 2007; Kharrat et al, 2010).

Thus, they introduce the kernel function in Equation (8) and they obtain the following function of classification:

$$
\begin{aligned}
& k\left(\vec{x}_{i}, \vec{x}_{j}\right)=!\left(\overrightarrow{x_{i}}\right) \cdot !\left(\vec{x}_{j}\right) \\
& f(\vec{x})=\sum_{i=1}^{N} \alpha_{i} y_{i} K\left(\vec{x}_{i}, \vec{x}_{j}\right)+b
\end{aligned}
$$

With: 


$$
k(\vec{x}, x)=\exp \left(-\frac{\vec{x}-\vec{x}^{2}}{2 \alpha^{2}}\right)
$$

Several kernel and parameters are tested to select the best one (Parveen et al, 2015).

\section{Sequential Minimal Optimization (SMO)}

Sequential Minimal Optimization Algorithm (SMO) is an algorithm that solves quickly the quadratic problem (Ramakrishnan et al, 2017) of SVM without going through all the steps of numerical resolution. The main idea of decomposition algorithms is to work with a subset of data that reduces the problem, keep the solutions and continue with the rest of the data where previous solutions have yet to be tested. The SMO takes this idea to the extreme: it optimizes only two vectors by iteration. This optimization admits an analytical solution. At each iteration, the SMO chooses two Lagrange coefficients $\alpha_{i}$ and $\alpha_{j}$ to optimize together, finding optimal values since all the others are fixed and update the solution vector $\alpha$.

$$
\begin{aligned}
& L D=\alpha_{1}+\alpha_{2}-\frac{1}{2} k\left(x_{1} \cdot x_{1}\right) \alpha_{1}^{2}-\frac{1}{2} k\left(x_{2} \cdot x_{2}\right) \alpha_{2}^{2}-2 y_{1} y_{2} k\left(x_{1} \cdot x_{2}\right) \alpha_{1} \alpha-y_{1} \alpha_{1} v_{1}-y_{2} \alpha_{2} v_{2}+\text { cte } \\
& v_{i}=\sum_{j=3}^{l} y_{i} \alpha_{i} k\left(x_{i} \cdot x_{j}\right)
\end{aligned}
$$

Where

$$
0 \leq \alpha_{1}, \alpha_{2} \leq \text { cte and } \sum_{i=1}^{l} \alpha_{i} y_{i}=0
$$

Error classification is defined by:

$$
\begin{aligned}
& \alpha_{2}^{\text {new }}=\alpha_{2}^{\text {old }}-\frac{y_{2}\left(E_{1}-E_{2}\right)}{k} \\
& \alpha_{2}^{\text {new }}=\alpha_{2}^{\text {new }}+y_{1} y_{2}\left(\alpha_{2}^{\text {old }}-\alpha_{2}^{\text {new }}\right)
\end{aligned}
$$

with:

$$
k=k\left(x_{1} \cdot x_{1}\right)+k\left(x_{2} \cdot x_{2}\right)-2 k\left(x_{1} \cdot x_{2}\right)
$$


The stopping criterion of the SMO must verify the KKT conditions [Ta-Wen Kuan et al, 2001].

\section{EXPERIMENTAL RESULTS}

The proposed method is implemented in $\mathrm{C}++$ builder. The authors have conducted their experiments on real weighted MRI T1 and T2 images of patients with multiple sclerosis. The images were obtained using a matrix of size $181 \mathrm{X} 181$, number of slices $=20$ and thickness slices $=5 \mathrm{~mm}$.

The Figures 2, 3, 4 and 5 show the obtained results of AIS approach. Figures 2(1), 3(1), 4(1), and 5(1) show the original images, Figures 2(2), 2(3), 2(4); 3(2), 3(3), 3(4), 4(2), 4(3), 4(4) and 5(2), 5(3), 5(4) show the AIS results and finally Figure 2(5), 3(5), 4(5) and 5(5) Show the detected lesions.

They have compared the proposed approach with their previous approach (Merzoug et al, 2014) and another method which segments multiple sclerosis (MS) lesions in WM using a modified algorithm that relies on the histo based fast fuzzy C-means (HFFCM) (Yaswanth et al, 2016) using 3D simulated MR data (brainweb).

Table 1 shows the results of a comparison between the proposed approach and other techniques for detecting multiple sclerosis lesions.

The set detected voxels as lesions by the automated process are denoted by $\mathrm{S}$ and in the "ground truth" expert reference by R. True positive (TP) voxels are the voxels common to both S and R. True negative (TN) voxels are all voxels not outlined as lesions by experts. False positives (FP) are those detected in $\mathrm{S}$ but not by $\mathrm{R}$ and false negatives (FN) are those identified in $\mathrm{R}$ but not in $\mathrm{S}$.

In order to analyze the robustness of the proposed approach, they have used the following criteria:

Sensitivity SE: TP/(TP + FN)

Specificity SP: TN/(TN + FP)

Accuracy AC: $(\mathrm{TN}+\mathrm{TP}) /(\mathrm{TN}+\mathrm{TP}+\mathrm{FN}+\mathrm{FP})$

The different results show generally that the proposed method provides better results. For the proposed method, the researchers show that they have obtained high sensitivity, it means that there are few false negative results, and then the fewer cases of disease are missed. However the obtained values of specificity of their method are slightly smaller than the obtained values of Yaswants (Yaswanth et al, 2016) but the results mean that there are few false positive results.

\section{CONCLUSION}

In this paper, the researchers have proposed an approach of multiple sclerosis lesions segmentation in 3D brain MR images based on three steps. The first one segments brain tissue by artificial immune systems (AIS). Then the second step extracts features and the last one classifies MS lesions by SVM.

For further research, the authors can propose to use another features to remove irrelevant and redundant features, and to conduct the experiments on other brain database IRM. Also one could improve the detection of lesions in MS by exploring the techniques based on Fuzzy neural networks. 
Figure 2. (1) Original brain image, (2, 3, 4) segmented image by AIS (brain tissues), (5) MS lesions detected by SVM

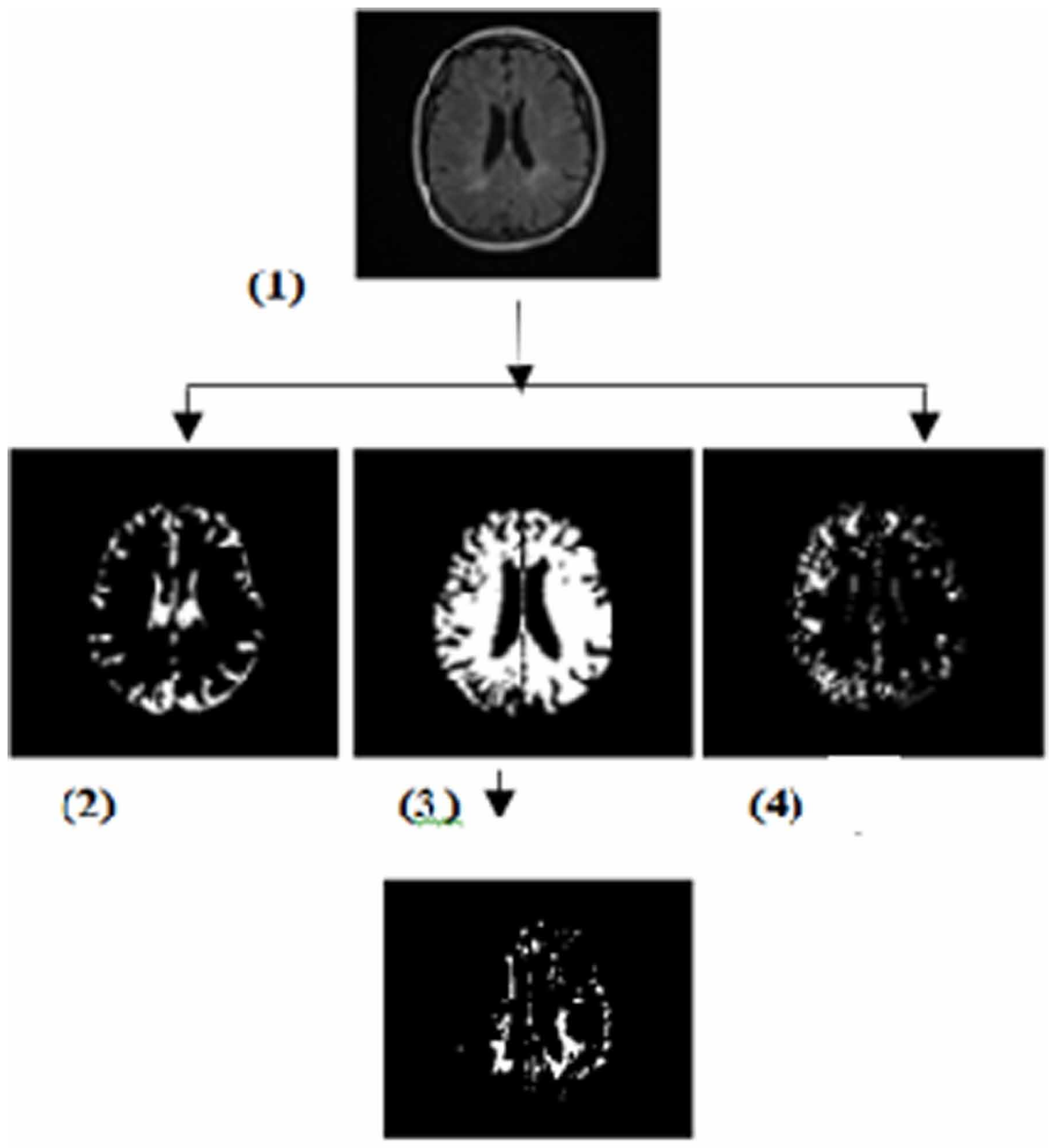

(5) 
International Journal of Cognitive Informatics and Natural Intelligence

Volume 15 • Issue 2 • April-June 2021

Figure 3. (1) Original brain image, $(2,3,4)$ segmented image by AIS (brain tissues), (5) MS lesions detected by SVM

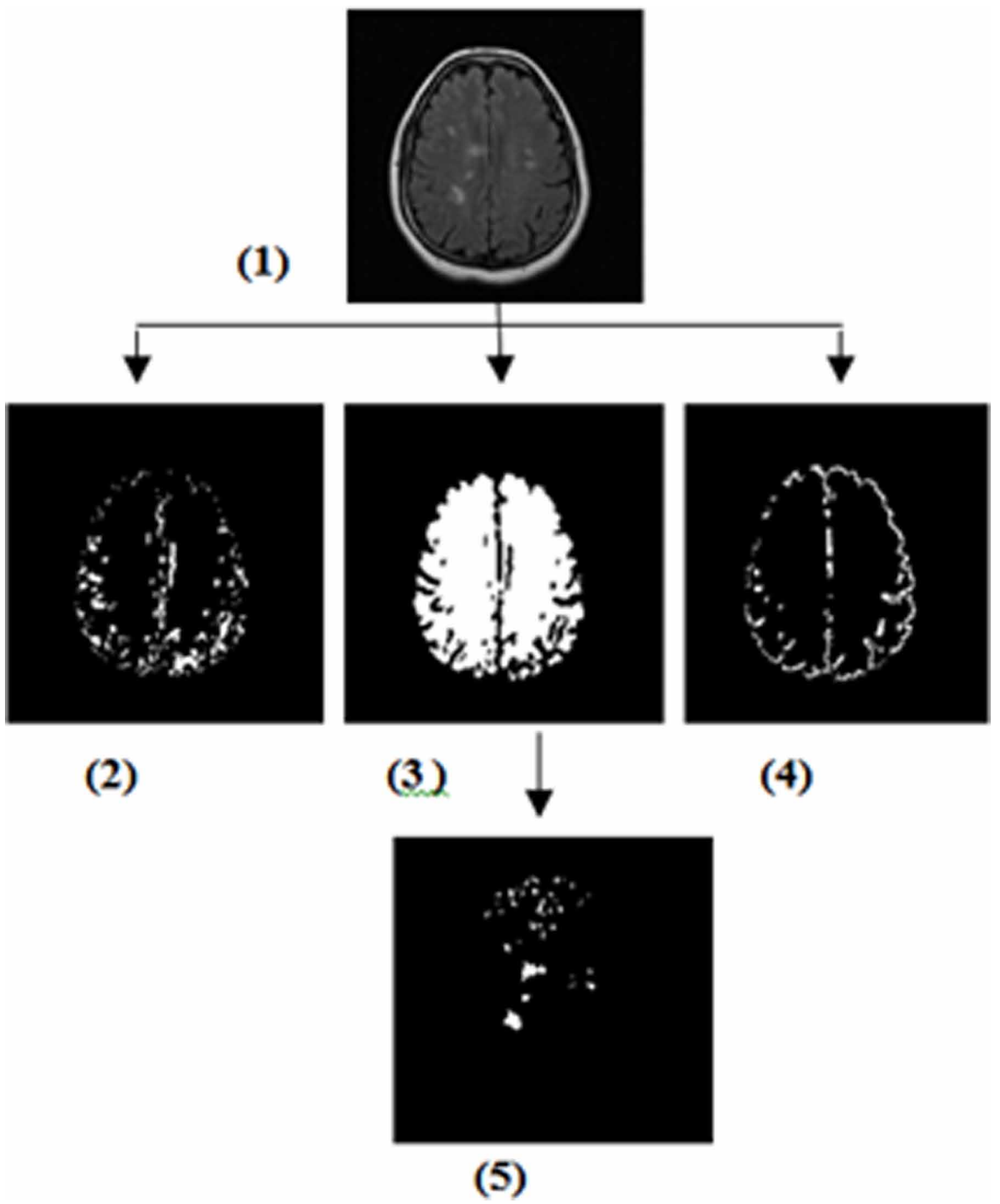


Figure 4. (1) Original brain image, $(2,3,4)$ segmentation results by AIS (brain tissues), (5) MS lesions detected by SVM

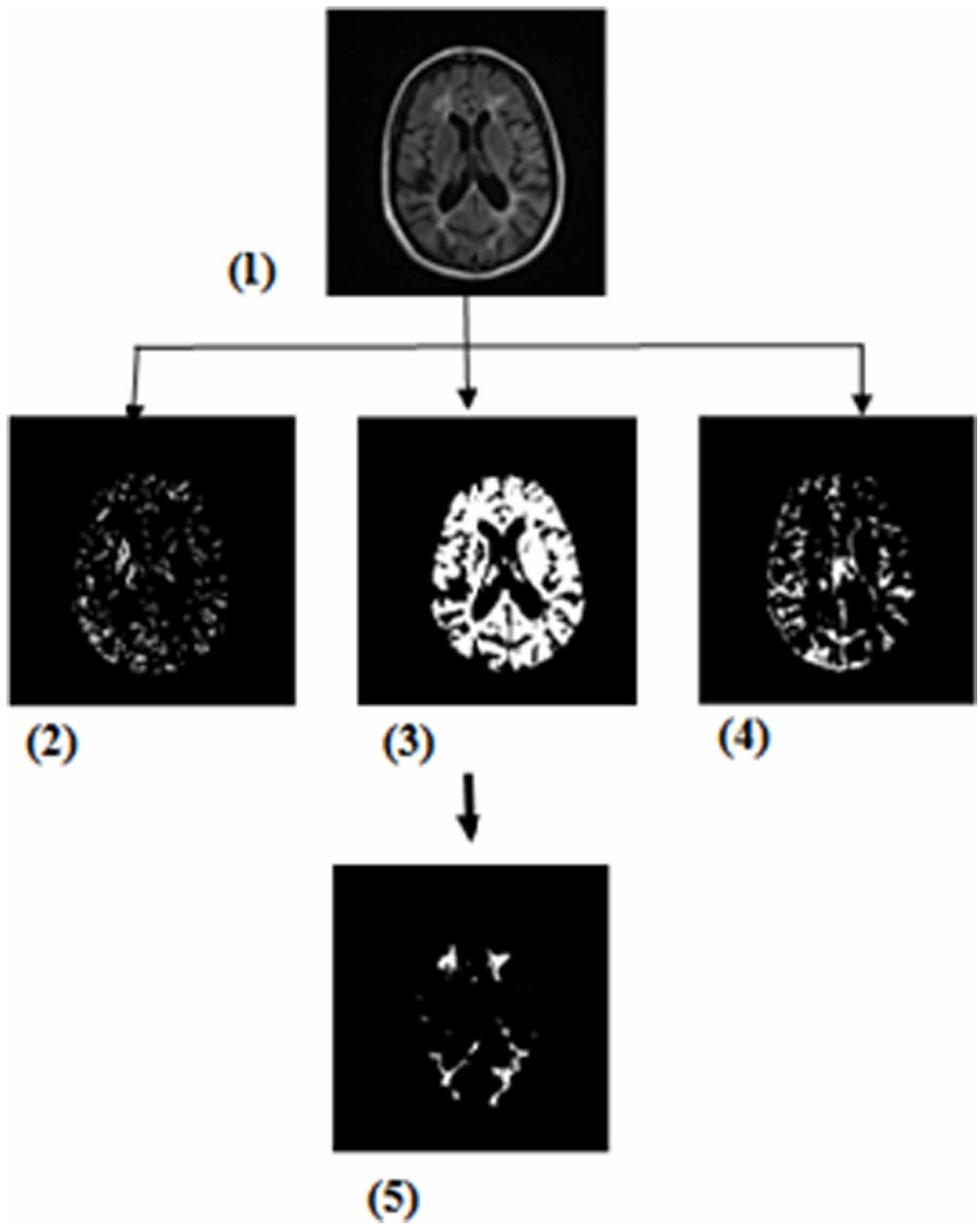


Figure 5. (1) Original brain image, $(2,3,4)$ segmentation results by AIS (brain tissues), (5) MS lesions detected by SVM

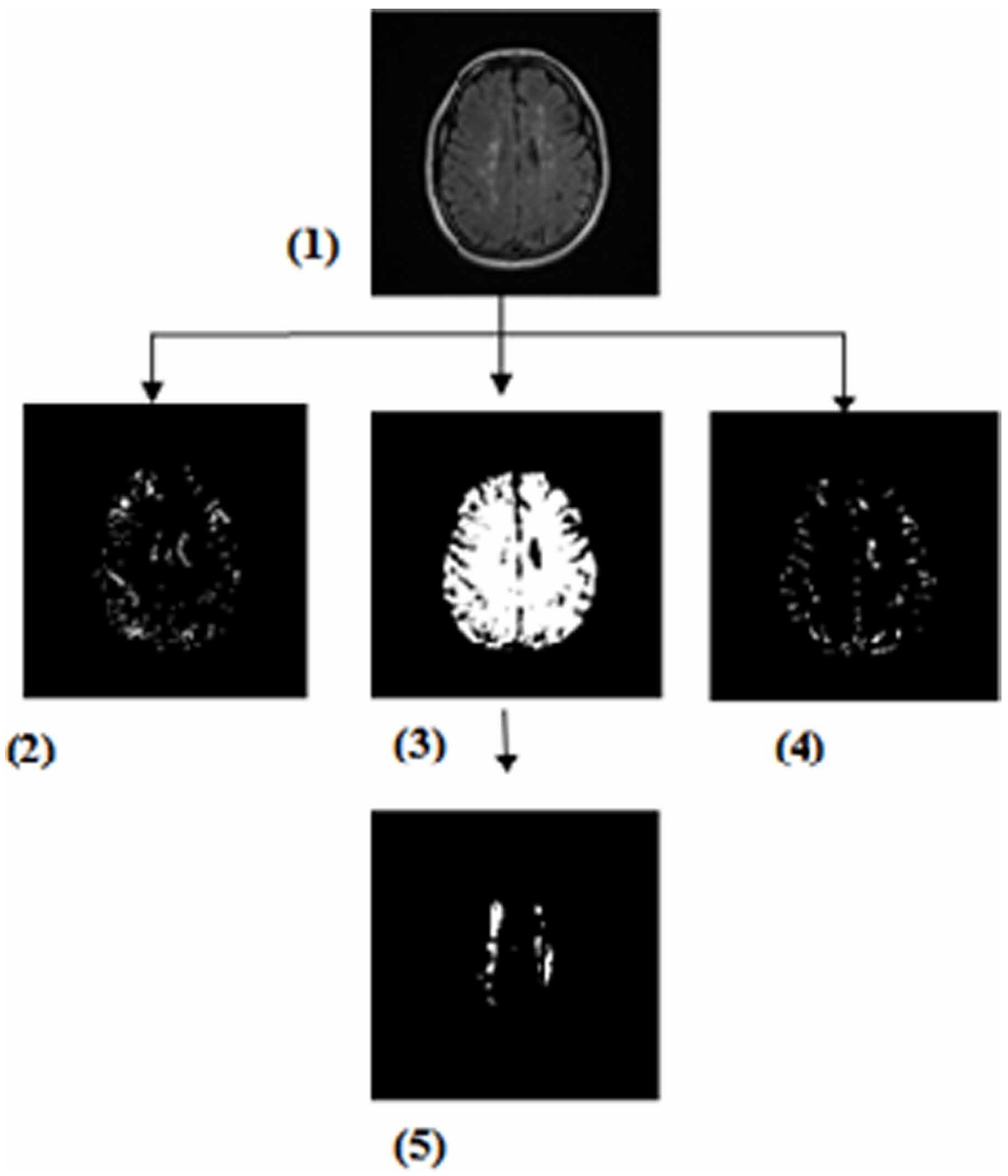

Table 1. Comparison of SE, SP and AC measures

\begin{tabular}{|l|l|l|l|l|l|l|l|l|}
\hline \multicolumn{4}{|c|}{ The Proposed Method } & \multicolumn{3}{c|}{ [Merzoug et al, 2014] } & \multicolumn{3}{c|}{ [Yaswanth et al, 2016] } \\
\hline \multicolumn{1}{|c|}{ SE } & \multicolumn{1}{|c|}{ SP } & \multicolumn{1}{|c|}{ AC } & SE & SP & AC & SE & SP & AC \\
\hline 0.99 & 0.895 & 0.996 & 0.997 & 0.118 & 0.992 & 0.258 & 0.993 & 0.959 \\
\hline 1 & 0.838 & 0.998 & 0.991 & 0.475 & 0.988 & 0.318 & 0.996 & 0.955 \\
\hline 1 & 0.789 & 0.997 & 0.989 & 0.309 & 0.984 & 0.433 & 0.994 & 0.965 \\
\hline
\end{tabular}




\section{REFERENCES}

Abdelhadi, A., Mouss, H., \& Kadr, I. O. (2010). Algorithme Du Système Immunitaire Artificiel P La Surveillance Industrielle. In International conference on industrial engineering and manufacturing ICIEM'10. Batna.

Akselrod-Ballin. (2009). Automatic Segmentation and Classification of Multiple Sclérosis in Multichannel MRI. IEEE Transactions on Biomédicale Engineering.

Bassem Abdullah, A. (2012). Segmentation of Multiple Sclerosis Lesions in Brain MRI. Open access Dissertations 711. University of Miami, Scholarly Repository.

Belmokhtar, N., \& Benamrane, N. (2012). Classification of Alzheimer's Disease from 3D Structural MRI Data. International Journal of Computers and Applications, 47(3), 40-44. doi:10.5120/7171-9798

Chandan, S. J., \& Sujatha, B. K. (2018). Automatic Segmentation of Tumor Regions in Brian MRI Images and Classification of Various Stages. International Journal of Electronics. Electrical and Computational System IJEECS ISSN, 7(1), 2348-117X.

Cuevas, E., Osuna-Enciso, V., Zaldivar, D., Pérez-Cisneros, M., \& Sossa, H. (2012). Multithreshold Segmentation Based on Artificial Immune Systems. Mathematical Problems in Engineering, 2012(874761), 20. doi:10.1155/2012/874761

Faro, A., Giordano, D., Spampinato, C., \& Pennisi, M. (2010). Statistical Texture Analysis of MRI Images to Classify Patients Affected by Multiple Sclerosis. XII Mediterranean Conference on Medical and Biological Engineering and Computing 2010. IFMBE Proceedings, 29. doi:10.1007/978-3-642-13039-7_68

Gheshlagh, Madani, Suratgar, \& Faraji. (2018). Segmentation Of Multiple Sclerosis Lesion In Brain Mr Images Using Fuzzy C-Means. [International Journal of Artificial Intelligence and Applications, 9, 2.

Hassan, K., \& Afrasiabi, M. (2011). Segmentation of Multiple Sclerosis Lesions in Brain MR Images Using Spatially Constrained Possibilistic Fuzzy C-Means Classification. Journal of Medical Signals and Sensors, l(3), 149-155. PMID:22606670

Jéré, L., \& Christian, B. (2007). Segmantation d'Images Cérébrales: Etat de l'art. Institut National de Recherche en Informatique et en Automatique, Rapport de recherche, No. 6306 - pp.46, Version 3.

Kharrat, A., Gasmi, K., Ben Messaoud, MB., Benamrane, N., Abid, M.(2010). A hybrid approach for automatic classification of brain MRI using genetic algorithm and Support Vector Machine. Leonardo Journa of Sciences, $71-82$

Komaki, G. M., Teymourian, E., \& Kayvanfar, V. (2016). Minimising Makespan In The Twostage Assembly Hybrid Flow Shop Scheduling Problem Using Artificial Immune Systems. International Journal of Production Research, 54(4), 963-983. doi:10.1080/00207543.2015.1035815

Kuan, T.-W., Wang, J.-F., Wang, J.-C., Lin, P.-C., \& Gu, G.-H. (2001). VLSI Design of an SVM Learning Core on Sequential Minimal Optimization Algorithm. IEEE Transactions on Very Large Scale Integration (VLSI) Systems, 20, 4.

Lladó, X., Oliver, A., Cabezas, M., Freixenet, J., Quiles, A., Valls, L., Ramió-Torrent, L., \& Rovira, À. (2012). Segmentation Of Multiple Sclerosis Lesions In Brain MRI: A Review Of Automated Approaches. Information Sciences, 186(1), 164-185. doi:10.1016/j.ins.2011.10.011

Madjid, T., Kazemi, M. R., Vafadarnikjoo, A., \& Mobin, M. (2016). An Artificial Immune Algorithm For Ergonomic Product Classification Using Anthropometric Measurements. Elsevier.

Merzoug, A., Benamrane, N., \& Taleb-Ahmed, A. (2014). MS lesions segmentation in 3D MR images using FCM and SVM. 4th International Conference on Image Processing Theory, Tools and Applications (IPTA), 29-33. doi:10.1109/IPTA.2014.7001924

Parveen \& Singh. (2015). Detection of Brain Tumor in MRI Images, using Combination of Fuzzy C-Means and SVM. In 2nd International Conference on Signal Processing and Integrated Networks (SPIN). IEEE.

Ramakrishnan, T., \& Sankaragomathi, B. (2017). A Professional Estimate On The Computed Tomography Brain Tumor Images Using SVM-SMO For Classification And MRG-GWO For Segmentation. Pattern Recognition Letters, 94(C), 163-171. doi:10.1016/j.patrec.2017.03.026 
Rode, K. N., \& Patil, R. T. (2012). Texture Analysis Of Mri Using Svm \& Ann For Multiple Sclerosis Patients. International Journal of Engineering Research and Applications, 2, 4.

Urien, H., Buvat, I., Rougon, N., \& Bloch, I. (2016). A 3D Hierarchical Multimodal Detection And Segmentation Method For Multiple Sclerosis Lesions In MRI. Proceedings of the 1st MICCAI Challenge on Multiple Sclerosis Lesions Segmentation Challenge Using a Data Management and Processing Infrastructure - MICCAI-MSSEG, 69-73.

Vera-Olmos, F., Melero, H., \& Malpica, N. (2016). Random Forest for Multiple Sclerosis Lesion Segmentation. Proceedings of the 1st MICCAI Challenge on Multiple Sclerosis Lesions Segmentation Challenge Using a Data Management and Processing Infrastructure - MICCAI-MSSEG, 81-86.

Veronese, E., Calabrese, M., Favaretto, A., Gallo, P., Bertoldo, A., \& Grisan, E. (2013). Automatic Segmentation of Gray Matter Multiple Sclerosis Lesions on DIR Images. In XIII Mediterranean Conference on Medical and Biological Engineering and Computing. Springer International Publishing.

Yaswanth Bhanumurthy, M., \& Koteswararao, A. (2016). An Automated MRI Segmentation Framework for Brains with Tumors and Multiple Sclerosis Lesions. In International Conference on Computation of Power, Energy Information and Communication (ICCPEIC). IEEE. doi:10.1109/ICCPEIC.2016.7557201

Zidelmal Amirou, Z., Amirou, A., Djeddi, M., \& Djouaher, N. (2007). Application des SVMs basées sur l'algorithme SMO pour la détection des anomalies cardiaques. 4th International Conference: Science of Electronic, Tehnologies of Information and Telecommunications.

Amina Merzoug is a PhD student. She obtained her master degree in Imaging and computer vision from University of Science and Technology of Oran Mohamed Boudia. Her research interests include image processing and medical imaging.

Nacéra Benamrane is currently a full professor and a director of SIMPA laboratory in department of Computer science at University of Science and Technology of Oran-Mohamed Boudiaf (USTO-MB). She received her engineering degree in Computer Science from University of Oran, the M.Sc. and Ph.D. degrees from University of Valenciennes, France. Since 2002, she is the head of vision and medical imaging team at SIMPA laboratory. She has published more than 100 papers in journals and conference proceedings. Her main research interests include image processing, medical imaging, computer vision, biomedical engineering and pattern recognition.

Abdelmalik Taleb-Ahmed received the Maitrise and DEA degrees in electronics in 1987 and 1989, respectively, and the Doctorate degree in signal processing and image processing from the Universit'e des Sciences et Technologie de Lille, France, in 1992. From 1992 to 2004, he was a Professor at the Universit'e des Sciences et Technologie de Lille. He is currently a full Professor with IEMN, Polytechnic University of Hauts-de-France. His research interests include digital signal processing and biomedical image processing. 